\title{
Installation des jeunes agriculteurs et pluriactivité au Costa Rica
}

\author{
Mathilde Parquet ${ }^{1, *}$ et Jean-François Le $\mathrm{Coq}^{2,3}$ \\ ${ }^{1}$ École normale supérieure de Lyon, 15, parvis René-Descartes, BP 7000, 69342 Lyon cedex 07, France \\ 2 Cirad, UMR Art-Dev, 34398 Montpellier, France \\ ${ }^{3}$ Centro Internacional de Agricultura Tropical (CIAT), Departamento Análisis de Políticas (DAPA), Cali, Colombie
}

\begin{abstract}
Résumé - Depuis quelques années, la petite agriculture familiale est mise sur le devant de la scène par les institutions internationales et présentée comme étant susceptible de répondre aux enjeux globaux (sécurité alimentaire et changement climatique), et ce, notamment, dans les pays du Sud où ce type d'agriculture reste majoritaire. Un groupe d'acteurs clés est toutefois oublié des études agricoles : les jeunes agriculteurs, qui sont pourtant de potentiels innovateurs et qui assurent le relais entre les générations et le maintien de ces agricultures. Cet article étudie les processus d'installation des jeunes agriculteurs familiaux au Costa Rica, dans deux communautés issues de réformes agraires. Nous montrons qu'un important changement social est à l'œuvre avec la montée du travail à temps partiel dans l'activité agricole. Nous mettons en évidence l'existence de trois types d'installation distincts que nous qualifions de relève, reprise et création. Alors que la reprise et la création génèrent des changements dans l'exploitation et dans l'activité agricole, la relève, plus fréquente, n'entraîne aucun changement significatif. Les facteurs explicatifs sont à chercher du côté de logiques territoriales, de dotation en capitaux différenciés et de la relation avec l'ancien chef d'exploitation qui est souvent le père. Notre analyse souligne l'importance de la prise en compte de la diversité des formes d'installation pour promouvoir des politiques adaptées facilitant le renouvellement générationnel dans l'agriculture familiale dans les pays du Sud.
\end{abstract}

Mots clés : jeunes agriculteurs / installation / agriculture familiale / petite agriculture / Costa Rica

\begin{abstract}
Settling of young farmers and pluriactivity in Costa Rica. Small-scale family agriculture has been encouraged for several years by international institutions as an answer to global issues (food security and climate change), especially in Southern countries where this type of agriculture still prevails. One group of key actors is still poorly analyzed in agricultural studies: the young farmers. Yet they are potential innovators, they insure the generational renewal and thus maintain family agriculture. This paper studies the settling process of young farmers in two communities in Costa Rica resulting from land reforms. We show an important social change with the development of part-time farming activity. Three types of settling are identified: the taking over, the resumption and the creation of a new farm. Whereas resumption and creation trigger changes in the farm and in agriculture, taking over, the most common case, does not give rise to change. Territory characteristics, different capital endowment and the relationship to the former farm owner, who is often the father, can explain these different types of settlings. Our analysis highlights the necessity to consider the diversity of settling processes to promote adapted policy aiming at facilitating the generational renewal in family agriculture.
\end{abstract}

Keywords: young farmers / settling / family agriculture / small-scale agriculture / Costa Rica

\section{Introduction}

Alors que l'agriculture familiale est promue comme une solution de développement durable, notamment dans les pays du Sud (Sourisseau, 2014 ; Lacombe, 2016), l'apport des jeunes agriculteurs y reste peu étudié (Amichi et al., 2015).

\footnotetext{
* Auteur de correspondance : mathilde.parquet@ens-lyon.fr
}

Il est pourtant crucial de s'y intéresser pour comprendre la diversité des agricultures familiales et ses évolutions à moyen terme (Desmulier et Gasselin, 2015) et pour construire des politiques publiques adaptées à la diversité des situations des agricultures familiales (Sabourin et al., 2014). En effet, dans le contexte d'urbanisation et de tertiarisation des économies, les pays du Sud font face à une dépréciation du métier d'agriculteur, à un vieillissement des chefs d'exploitation et à un délaissement de l'agriculture par les jeunes générations 
Tableau 1. Caractéristiques historiques et socio-économiques des zones d'étude.

Table 1. Historical and socio-economical characteristics of study areas.

\begin{tabular}{|c|c|c|}
\hline & Valle Azul & Futuro \\
\hline \multirow[t]{3}{*}{ Date de création et caractéristiques de l'asentamiento ${ }^{\mathrm{a}}$} & Fondé en 1978 & Fondé en 1991 \\
\hline & 115 familles bénéficiaires & 110 familles bénéficiaires \\
\hline & Parcelles d'environ 9 ha & Parcelles d'environ 4 ha \\
\hline Visibilité du parcellaire hérité de la réforme agraire ${ }^{b}$ & Non & Oui \\
\hline Nombre d'habitants en $2012^{c}$ & 926 & 703 \\
\hline Nombres de foyers en 2012 (viviendas) $^{\mathrm{c}}$ & 401 & 214 \\
\hline Infrastructures $^{\mathrm{a}}$ & $\begin{array}{l}\text { Salle des fêtes, maternelle, école primaire, } \\
\text { collège technique, centre de santé }\end{array}$ & Salle des fêtes, école primaire \\
\hline Activités $^{\mathrm{a}}$ & $\begin{array}{l}\text { Agriculture, petit artisanat (ébénisterie, } \\
\text { recyclage), hébergements touristiques }\end{array}$ & $100 \%$ agriculture \\
\hline
\end{tabular}

\footnotetext{
${ }^{\mathrm{a}}$ Entretiens auprès des acteurs de la zone.

${ }^{\mathrm{b}}$ Observations des auteurs en 2015.

${ }^{\mathrm{c}}$ Données des centres de santé de Valle Azul et Futuro, 2012.
}

(FAO et al., 2014). S'intéresser à la catégorie des jeunes agriculteurs, c'est s'intéresser à ceux qui, dans un tel contexte, assurent, perpétuent ou font évoluer cette agriculture familiale. Dans les pays occidentaux, cette catégorie a été initialement étudiée par les historiens et a été saisie comme facteur clé de modernisation agricole (Duby et al., 1992). D'autres travaux plus récents se sont intéressés aux jeunes exploitants par le prisme des organisations collectives (Purseigle, 2004) et des politiques dont ils sont l'objet comme par exemple, en France, la dotation jeunes agriculteurs (DJA) (Mundler et Ponchelet, 1999 ; Le Blanc, 2011). Le traitement de la question des jeunes agriculteurs dans les pays du Sud est plus récent (Chinsinga et Chasukwa, 2012). Les études concernent principalement le continent africain, et en particulier le Maghreb. À notre connaissance, seuls deux articles récents portent sur l'Amérique latine (Coradini, 2015 ; Costa et Ralisch, 2013). Ces travaux envisagent les jeunes exploitants soit en tant qu'objets de politiques agricoles (Rémy, 1997 ; Muhammad-Lawal et al., 2003), soit à travers leurs modalités d'accès aux ressources productives, particulièrement à l'eau et à la terre (Hamamouche et al., 2015), soit en rapport avec les problématiques d'emploi des jeunes ruraux (Davis, 2008 ; White, 2012) et d'émigration (Bezu et Holden, 2014 ; Bednaríkova et al., 2016). Toutefois, le processus d'installation même des jeunes agriculteurs n'est pas étudié, alors qu'il s'agit d'un point crucial pour comprendre les évolutions des agricultures du Sud et le processus de renouveau générationnel dans les agricultures familiales.

Nous nous proposons donc de caractériser la diversité des processus d'installation des jeunes agriculteurs et d'identifier les facteurs explicatifs de cette diversité à partir d'une étude de terrain conduite dans la région Nord du Costa Rica, où l'agriculture familiale constitue encore l'essentiel des exploitations (Salazar et al., 2012). Nous faisons l'hypothèse que la nature et l'ampleur des changements agricoles liés à l'installation de jeunes agriculteurs dépendent de leur processus d'installation, de leur dotation en capitaux et de leur degré d'autonomie et d'indépendance vis-à-vis du chef d'exploitation de la génération précédente.

Après avoir présenté la méthode utilisée, nous analyserons les différentes formes d'installation et mettrons en évidence trois types d'installation aux caractéristiques distinctes, que nous qualifierons de création d'exploitation agricole, de relève et de reprise. Puis, nous identifierons les facteurs explicatifs de la diversité de ces processus d'installation. Enfin, nous discuterons des résultats de notre étude à la lumière des études récentes conduites dans d'autres contextes.

\section{Terrains d'étude et méthodologie}

\subsection{Terrains d'étude}

Notre étude a été réalisée au Costa Rica, un pays d'Amérique centrale qui a connu au cours des deux dernières décennies un rapide développement économique. Si l'agriculture y représente encore aujourd'hui $6 \%$ du produit intérieur brut et $37 \%$ des exportations, son importance ne cesse de diminuer au profit du secteur tertiaire, dont le développement se concentre autour de la capitale et attire les jeunes ruraux (Mesen, 2009).

Si de grandes exploitations de type agrobusiness dominent la production tournée vers l'exportation (banane), l'agriculture familiale occupe toujours une place importante dans certaines filières telles que le café, les cultures alimentaires ou l'élevage, notamment au nord du pays (Faure et al., 2010). Dans cette région, l'agriculture familiale se situe majoritairement dans les espaces de réforme agraire, où les terres de grandes exploitations ont été redistribuées aux paysans sans terre à partir des années 1970, donnant lieu à des communautés nommées asentamientos (Faure et Samper, 2005).

Notre étude analyse les processus d'installation des jeunes agriculteurs familiaux dans deux communautés issues de la réforme agraire : les asentamientos de Valle Azul et du Futuro (Tab. 1). Ces deux communautés présentent des caractéristiques géographiques, agro-écologiques et économiques similaires. Elles sont relativement proches des pôles d'attraction économique que sont la capitale, San José, et la zone touristique de La Fortuna (Fig. 1) et le prix du foncier y est relativement élevé du fait d'une forte demande des classes moyennes supérieures en quête de campagne pour des usages récréatifs. Elles combinent élevage bovin extensif et petite agriculture commerciale (plantes ornementales, manioc, patate douce ou taro) orientée vers l'exportation ou le marché national (Ruben et Saenz, 2008). 

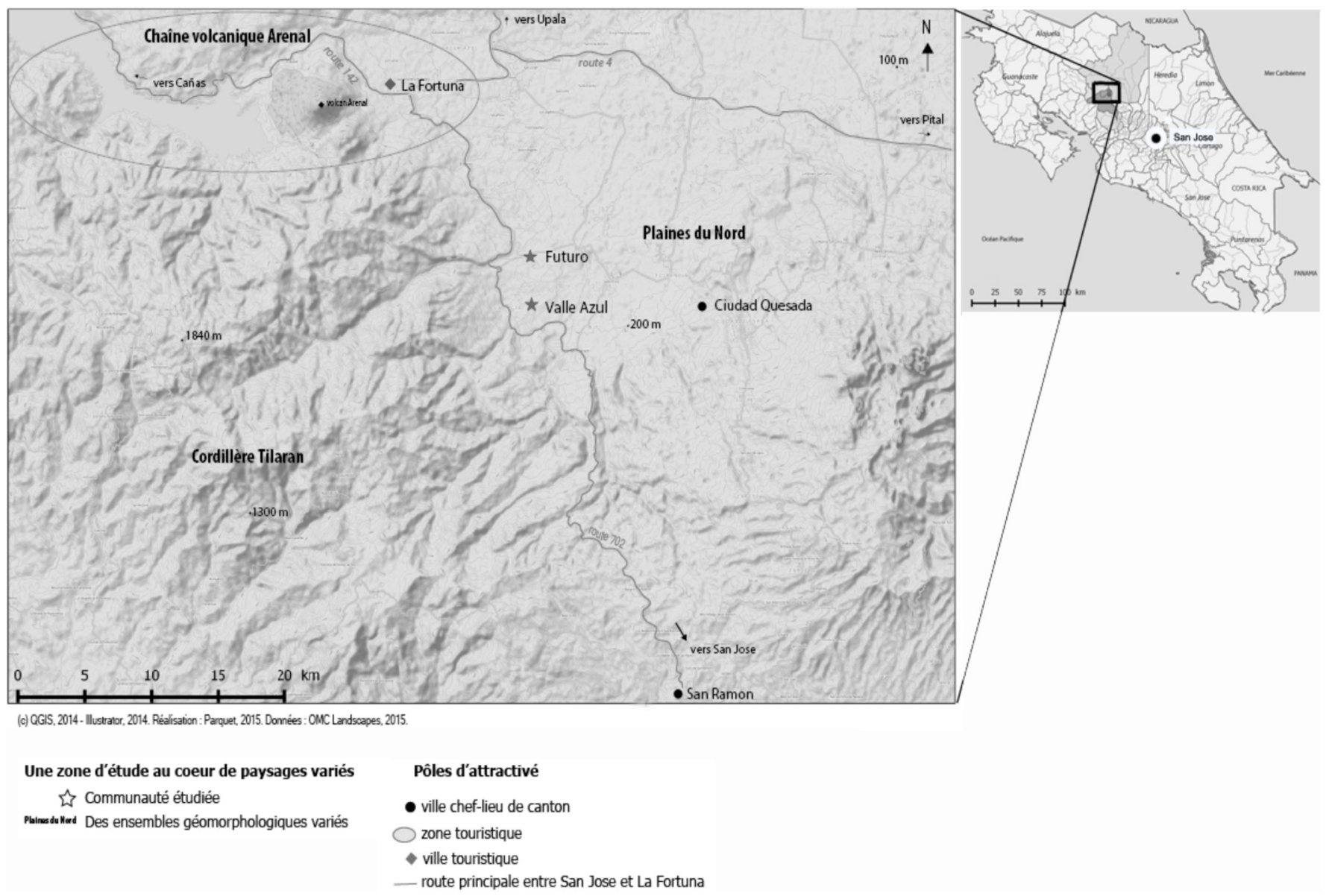

Fig. 1. Localisation régionale de la zone d'étude.

Fig. 1. Regional localization of the study area.

Toutefois, les situations foncières et les dynamiques de développement y sont différentes. La création de l'asentamiento de Valle Azul date de 1978, celle de Futuro date de 1990. Le parcellaire égalitaire de la réforme agraire n'est plus visible à Valle Azul, alors qu'il reste parfaitement identifiable à Futuro. En outre, les infrastructures (collège et centre de santé notamment) sont plus nombreuses et les activités économiques plus diversifiées à Valle Azul, alors que les infrastructures restent minimes à Futuro (église, salle des fêtes et école primaire seulement) et l'agriculture y est toujours l'activité quasi exclusive (Tab. 1).

\subsection{Méthodologie}

Pour appréhender la diversité des stratégies mises en place par les agriculteurs familiaux (Sourisseau et al., 2012), ainsi que le profil des exploitations des jeunes agriculteurs, nous avons mobilisé l'approche livelihood system (Chambers et Conway, 1991) en caractérisant les différents capitaux des exploitations, à savoir les capitaux naturel, physique, financier, humain et social (Tab. 2). Nous avons conduit des entretiens semi-directifs auprès de 27 jeunes agriculteurs et 13 exploitants de la génération précédente, pour saisir l'évolution des exploitations (Tab. 3). La grille d'entretien appliquée aux jeunes agriculteurs comprenait trois parties. La première invitait l'agriculteur interviewé à raconter son installation dans
Tableau 2. Capitaux et indicateurs du livelihood system appliqués à la petite agriculture familiale costaricienne.

Table 2. Capitals and indicators of the livelihood system applied to the Costa Rican family small-scale agriculture.

\begin{tabular}{ll}
\hline Capitaux & Indicateurs \\
\hline $\begin{array}{l}\text { Capital } \\
\text { naturel }\end{array}$ & $\begin{array}{l}\text { Surface de l'exploitation } \\
\text { Présence d'arbres ou de forêts } \\
\text { Présence d'eau (source, ruisseau, rivière) }\end{array}$ \\
$\begin{array}{l}\text { Capital } \\
\text { physique }\end{array}$ & $\begin{array}{l}\text { Type et nombres d'outils (tracteurs, machettes, camions) } \\
\text { mécanisé) }\end{array}$ \\
$\begin{array}{l}\text { Capital type de système d'irrigation (gravitaire ou } \\
\text { financier }\end{array}$ & $\begin{array}{l}\text { Recours à l'autofinancement } \\
\text { Recours à un prêt bancaire }\end{array}$ \\
$\begin{array}{l}\text { Capital } \\
\text { humain }\end{array}$ & $\begin{array}{l}\text { Main-d'œunt auprès d'une connaissance } \\
\text { Main-d'œuvre contractuelle, intensité et type (salariat } \\
\text { ponctuel ou permanent) }\end{array}$ \\
& $\begin{array}{l}\text { Niveau d'études } \\
\text { Membre d'une organisation ayant trait à l'agriculture }\end{array}$ \\
$\begin{array}{l}\text { Capital } \\
\text { social }\end{array}$ & $\begin{array}{l}\text { Participation à des journées de formation agricole } \\
\text { Insertion dans des réseaux sociaux hors agricoles }\end{array}$
\end{tabular}

Sources : les auteurs ; d'après Sourisseau et al. (2012). 
Tableau 3. Répartition des entretiens réalisés par types et territoires. Table 3. Interviews carried out according the type of actors and the study area.

\begin{tabular}{lccc}
\hline & Valle Azul & Futuro & Total \\
\hline Jeune exploitant & 19 & 8 & 27 \\
Génération antérieure & 3 & 10 & 13 \\
Père du jeune exploitant qui & 1 & 6 & 7 \\
est dans le processus d'installation & & & \\
Exploitant actuel sans successeurs & 2 & 4 & 6 \\
Total & 22 & 20 & 44 \\
\hline
\end{tabular}

l'agriculture. La deuxième décrivait l'activité agricole actuelle et les changements par rapport à l'organisation de l'exploitation avant son installation, s'il y avait lieu. La troisième était prospective et concernait les évolutions souhaitées dans son activité.

Dans les travaux traitant des jeunes agriculteurs, la caractérisation de l'objet « jeune » pose souvent problème : elle est trop réductrice et ne révèle pas l'hétérogénéité des situations (Mesen, 2009). Certaines études prennent pour critère l'âge, sans pour autant trouver de consensus. Certains auteurs (Aggelopoulos et Arabatzis, 2010), en prenant comme référence les instruments de politique tels que la DJA, définissent un jeune agriculteur comme un homme de moins de 40 ans. Pour d'autres (Ameur et al., 2015), les jeunes agriculteurs sont la génération suivante, " les fils d'attributaires ». Depuis les travaux de Bourdieu (1984), la jeunesse est également perçue comme une construction sociale. Le critère peut être alors l'état d'esprit : les jeunes agriculteurs sont ceux qui visent à pratiquer une agriculture « moderne » (Quarouch et al., 2015). En retenant et en précisant les critères proposés par Bouzidi et al. (2015b), nous avons choisi de considérer les jeunes agriculteurs au sens de personnes récemment installées, peu importe leur âge, à la tête d'une exploitation agricole ; c'est-à-dire qu'ils opèrent les choix de gestion de l'unité de production, à la différence des jeunes qui travaillent comme ouvriers agricoles dans l'exploitation de leurs parents ou ailleurs. Par « récemment installés », nous avons considéré une période de cinq années, en nous inspirant des politiques de DJA. Bien qu'il n'existe pas de politique semblable au Costa Rica, ce critère nous paraît justifié dans la mesure où, comme en France, on peut estimer que les premières années d'installation sont cruciales et, qu'au-delà, l'organisation de l'exploitation est bien rodée.

Compte tenu des spécificités de notre terrain, où il n'existe ni registres de jeunes agriculteurs, ni politiques spécifiques qui leur sont destinées, nous avons élaboré l'échantillon de jeunes agriculteurs à interviewer sur la base des connaissances d'un jeune natif de Valle Azul, très impliqué dans la communauté. Puis, lors des entretiens, nous avons pratiqué la méthode dite « boule de neige » (Atkinson et Flint, 2010), jusqu'à couvrir tous les cas identifiés dans chaque zone d'étude. Nous avons finalement présenté la liste des jeunes agriculteurs faisant l'objet de l'enquête à l'antenne locale du ministère de l'Agriculture, qui n'a pas su nous indiquer d'autres personnes. Nous avons ainsi identifié au total 27 jeunes agriculteurs qui correspondaient à nos critères (Tab. 3). N'ayant pas retenu de critère d'âge pour définir les jeunes exploitants, l'âge des agriculteurs auprès de qui nous avons enquêté allait de 21 à 49 ans (moyenne : 34 ans).

\section{Renouveau des exploitations agricoles et différents types d'installation}

\subsection{Temps partiel et diversification des activités agricoles}

L'analyse des activités des jeunes exploitants met en lumière deux principaux changements dans la conduite des exploitations par rapport à la génération précédente. Le premier est le développement du temps partiel. Alors que tous les agriculteurs interrogés appartenant à la génération précédente pratiquaient l'agriculture à temps complet, une majorité des jeunes agriculteurs $(60 \%)$ se consacre à l'agriculture seulement à temps partiel (Tab. 4). Deux types de temps partiel ont été identifiés :

- des jeunes ayant une activité salariée stable et pour qui l'agriculture est une activité " récréative » permettant un complément et une diversification des revenus ;

- des jeunes pratiquant des activités précaires et pour qui

l'agriculture est «subie» et exercée pour permettre la subsistance du foyer.

Il faut toutefois souligner qu'il est assez difficile d'évaluer le temps consacré à l'activité agricole. Pour la majorité, l'activité agricole ne représente que quelques heures, avant ou après leur activité principale, ou quelques journées prises sur les week-ends.

Le second changement est l'évolution des systèmes de production vers davantage de diversification agricole. Alors que la génération précédente cultivait en moyenne 1,5 espèces différentes, les jeunes agriculteurs en cultivent en moyenne quatre. On note également un changement dans le type de culture : la patate douce, le manioc et les haricots ont remplacé le café ou la canne à sucre. Deux tendances s'observent en termes de diversification et de commercialisation (Tab. 5). Les jeunes disposant d'un capital humain (niveau d'étude) plus élevé s'orientent vers une production commerciale pour le marché international, alors que ceux qui ont des activités plus précaires cultivent des productions pour le marché local ou national.

\subsection{Trois processus d'installation : création, relève et reprise}

L'analyse des processus d'installation des jeunes agriculteurs auprès de qui nous avons enquêté permet d'identifier trois types d'installation que nous avons qualifiés de création, de relève et de reprise (Tab. 6). La création se différencie des autres types par le fait que le jeune débute une nouvelle exploitation. La création est souvent un moyen de mettre en place un projet nouveau. Le plus souvent, ce type d'installation se fait en s'associant avec un membre de la famille autre que le père (un oncle ou un beau-frère), qui a des capitaux complémentaires. Il est difficile d'évaluer l'ampleur des changements induits par ces installations puisqu'il n'y a pas de référent. Cependant, sur les sept cas de création analysés, deux concernaient une agriculture basée sur des principes de 
Tableau 4. Activités non agricoles et motivations pour la production agricole des jeunes agriculteurs à temps partiel.

Table 4. Non-farming jobs and motivation for farming production of part-time farmers.

\begin{tabular}{|c|c|c|c|}
\hline $\begin{array}{l}n=16(60 \% \text { des } \\
\text { jeunes agriculteurs })\end{array}$ & Type de poste occupé & Sécurité de l'emploi & Motivation à pratiquer l'agriculture \\
\hline Groupe $1(n=9)$ & $\begin{array}{l}\text { Agent touristique à La Fortuna, } \\
\text { contrôleur dans une usine } \\
\text { hydroélectrique, guide touristique, } \\
\text { assistant à l'université }\end{array}$ & $\begin{array}{l}\text { Emplois salariés stables, } \\
\text { (sécurité forte) }\end{array}$ & $\begin{array}{l}\text { Diversification des revenus familiaux. } \\
\text { Agriculture par choix pendant les } \\
\text { temps libres }\end{array}$ \\
\hline Groupe $2(n=7)$ & $\begin{array}{l}\text { Ouvrier agricole dans d'autres fermes, } \\
\text { ouvrier sur un chantier } \\
\text { hydroélectrique, garde de nuit, } \\
\text { chauffeur de taxi, homme à tout faire } \\
\text { (peinture, réparation, jardinier) }\end{array}$ & $\begin{array}{l}\text { Emplois précaires : } \\
\text { contrats à durée } \\
\text { déterminée ou sans } \\
\text { garantie de salaire } \\
\text { régulier }\end{array}$ & $\begin{array}{l}\text { Agriculture subie, intérêt pécuniaire, } \\
\text { n'aime pas l'agriculture mais l'exerce } \\
\text { pour cause de revenus insuffisants }\end{array}$ \\
\hline
\end{tabular}

Sources : entretiens, 2015.

Tableau 5. Type de production agricole, commercialisation et capitaux des jeunes agriculteurs.

Table 5. Kind of farming production, marketing and capitals of young farmers.

\begin{tabular}{lll}
\hline$n=23^{a}$ & Groupe $1(n=15)$ & Groupe 2 $(n=8)$ \\
\hline Produits cultivés & Plantes ornementales, manioc, patate & $\begin{array}{l}\text { Produits tropicaux traditionnels variés, } \\
\text { fruits }\end{array}$ \\
Commercialisation de la production & Exportation & Marché régional ou national \\
Recours à un ouvrier agricole de façon ponctuelle & Oui & Non \\
Dotation en capitaux & Moyenne à forte & Faible à moyenne \\
Capital naturel & Présence d'un point d'eau sur & Exploitations généralement moins bien \\
& l'exploitation & dotées \\
Capital financier & Autofinancement & Emprunt à des connaissances \\
Capital physique & Outils traditionnels, système & Outils traditionnels, parfois système \\
& d'irrigation mécanisé, location de & d'irrigation gravitaire \\
tracteurs pour les gros travaux, emploi & \\
ponctuel d'ouvriers agricoles & Moyen : pas d'affiliation à des réseaux & Faible : pas d'appartenance à des réseaux \\
napital social & formels agricoles mais participation à & nicoles, ni sociaux
\end{tabular}

\footnotetext{
${ }^{a}$ Quatre cas n'entrent dans aucun de ces deux groupes et n'en constituent pas un à part entière.
}

Sources : entretiens, 2015.

permaculture, rompant alors avec l'agriculture pratiquée dans la région. Les jeunes agriculteurs de ce type sont leur propre chef et jouissent d'une grande autonomie, comme en témoigne un jeune agriculteur de 23 ans installé avec un associé depuis un an à Valle Azul en louant un terrain à sa tante : « Ce qui me plaît [dans mon exploitation], c'est de travailler à mon compte, sans qu'il n'y ait personne pour me commander. "

Dans les processus de relève et de reprise, les jeunes agriculteurs se placent dans le sillon du chef d'exploitation précédent, le plus souvent le père. Bien que proches dans la langue française, nous considérerons ces deux termes pour désigner deux processus différents d'installation : la reprise est synonyme d'une relative autonomie de décision vis-à-vis du chef d'exploitation de la génération précédente, et la relève d'une faible autonomie de décision du jeune agriculteur.
La différence entre ces deux types se traduit aussi par l'ampleur de l'influence de l'installation du jeune agriculteur sur la trajectoire de l'exploitation.

Le type reprise est le plus rare (trois occurrences). Dans ce cas, c'est le jeune agriculteur qui prend toutes les décisions. Comme le dit le père d'un jeune agriculteur dont les fils viennent de reprendre l'exploitation : « Ce n'est pas moi qui travaille la propriété, c'est eux. Je travaille avec eux mais en réalité, c'est eux qui font tout. C'est eux qui prennent les décisions. » Les changements induits par ce type d'installation sont radicaux : réorganisation complète de l'exploitation avec introduction d'une activité d'élevage et de transformation afin de mieux valoriser la production, changement de types de culture, de débouchés commerciaux ou décision d'agrandir l'exploitation de façon notable. 
Tableau 6. Caractéristiques des différents types d'installation.

Table 6. Characteristics of the different kinds of settling.

\begin{tabular}{|c|c|c|c|}
\hline $\mathrm{n}=27$ & \multicolumn{3}{|c|}{ Types d'installation } \\
\hline Pourcentage de représentation & $27 \%$ & $62 \%$ & $11 \%$ \\
\hline $\begin{array}{l}\text { Pratique de l'agriculture à temps } \\
\text { partiel }\end{array}$ & $\begin{array}{l}\text { Minoritaire ( } 38 \% \text { des jeunes } \\
\text { avec ce type d'installation) }\end{array}$ & $\begin{array}{l}\text { Majoritaire (56\% des jeunes } \\
\text { agriculteurs avec ce type } \\
\text { d'installation) }\end{array}$ & $\begin{array}{l}\text { Très rare }(6 \% \text { des jeunes } \\
\text { agriculteurs avec ce type } \\
\text { d'installation })\end{array}$ \\
\hline
\end{tabular}

référence à l'exploitation antérieure

Caractéristique principale de

l'organisation de l'exploitation

Installation souvent avec un associé

\section{Autonomie décisionnelle}

Indépendance en termes de capitaux
Forte : le jeune prend l'ensemble des décisions concernant l'exploitation

Forte : le jeune est détenteur des moyens de production (terre achetée, louée ou héritée) et investit l'essentiel de la force de travail)

Date précise

Le jeune gère les revenus Partage des revenus entre associés.

Secondaire achevé En cours de formation
Le père ou l'ancien chef d'exploitation a encore un rôle. Relation et rôles entre le jeune et le père souvent complexes

Faible à moyenne : le père peut décider seul (avoir le dernier mot) ou considérer davantage l'avis du jeune

Moyenne : le père possède l'essentiel des moyens de production, mais le jeune dispose de quelques moyens de production propres (parcelle en héritage promis ou location, outils et matériels propres)

Souvent impossible de déterminer une date précise

Le père gère les revenus.

Participation à l'économie familiale avec ou sans séparation des revenus, mais non-monétarisation du travail

Supérieure à l'ancien chef d'exploitation

Primaire achevé
Le jeune est le nouveau chef d'exploitation. Le père s'en va ou occupe des tâches mineures sur l'exploitation

Forte : le jeune prend l'ensemble des décisions concernant l'exploitation

Faible : le père reste détenteur d'une partie des moyens de production (terre, outils) mais le jeune fournit l'essentiel de la force de travail, possède la terre (héritage acté, promis ou location) et les moyens de production

Date précise

Le jeune gère les revenus.

Participation à l'économie familiale mais non-monétarisation du travail familial

Supérieure à l'ancien chef d'exploitation

Souvent niveau universitaire

En cours de formation

Sources : entretiens, 2015.

Cependant, c'est la relève, le type d'installation générant le moins de changement, qui est majoritaire $(60 \%$ des cas observés). Le jeune agriculteur compose alors avec son père qui travaille toujours sur l'exploitation. Les relations pèresjeunes agriculteurs diffèrent toutefois. Dans les deux tiers des cas de relève analysés, les jeunes sont assez indépendants et développent des activités qui changent la trajectoire de l'exploitation. Le jeune peut ainsi développer des activités de commercialisation en achetant un camion afin de ne plus dépendre d'intermédiaires et d'accroître ses revenus. Le jeune peut également développer une activité non agricole complémentaire sur l'exploitation, qui diversifie les sources de revenu de la famille tout en les pérennisant. Par exemple, un jeune agriculteur a installé une salle de sport sur l'exploitation, dont il s'occupe le soir après le travail des champs, offrant ainsi un nouveau service aux habitants de la communauté qui n'avaient jusqu'alors pas accès à de telles infrastructures. Il s'agit alors d'une véritable collaboration entre le fils et le père et ces cas de relève peuvent se rapprocher de la reprise. Cependant, dans les cas de relève, le fils est toujours soumis au bon vouloir du père et n'a pas le dernier mot dans les décisions. Ainsi, dans un tiers des exploitations caractérisées par un processus de relève, le fils a peu de marge de manœuvre et, en conséquence, n'apporte aucun changement. Comme le rapporte un « jeune » agriculteur de 48 ans : « Oui nous prenons les décisions ensemble... mais papa a toujours le dernier mot. C'est lui [à 85 ans] qui travaille le plus... La semaine dernière papa a vendu deux de nos quatre bœufs... moi je voulais les garder... je suis d'accord que deux c'est assez, mais je n'avais pas envie de les vendre. »

Au-delà de cette caractérisation statique des types d'installation, nous avons observé des passerelles entre les types d'installation identifiés, qui peuvent témoigner d'une évolution vers davantage d'autonomie et d'indépendance des jeunes agriculteurs. Ainsi, la relève est souvent une forme initiale d'installation. L'évolution vers la reprise ou la création est majoritairement souhaitée par les jeunes agriculteurs. 


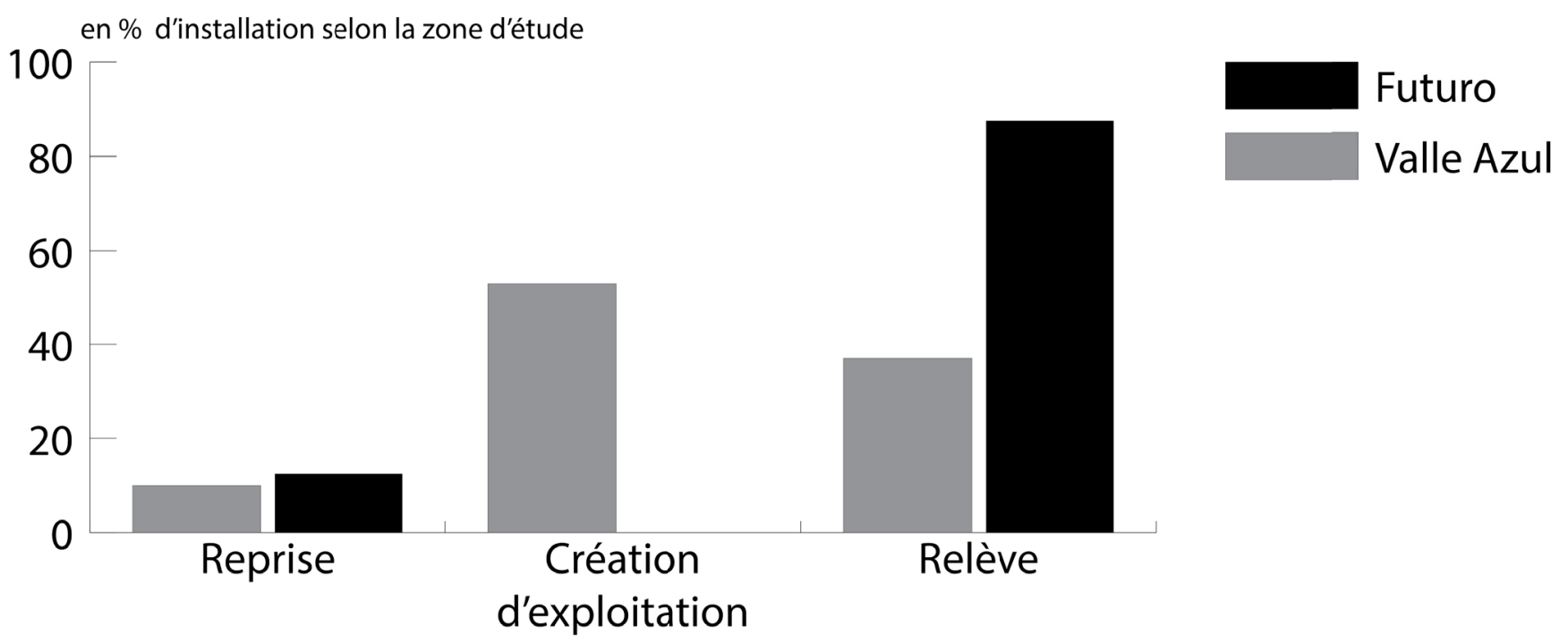

Fig. 2. Répartition des modalités d'installation par zone d'étude.

Fig. 2. Distribution of the different types of settling according to the study area.

Une autonomisation progressive peut alors mener vers une reprise (un cas observé). Toutefois, la relève n'est pas un passage obligé pour un jeune agriculteur de type reprise ou création.

\section{Facteurs explicatifs}

Trois variables permettent d'expliquer la diversité des types d'installation observés : la trajectoire des territoires, la dotation en capitaux et la relation au père.

\subsection{La trajectoire du territoire}

La dynamique d'installation est très contrastée entre les deux communautés (Fig. 2). De manière paradoxale, aucun cas de création n'a été rencontré à Futuro alors que ce territoire est plus agricole et que, à la différence de Valle Azul, une antenne du ministère de l'Agriculture, des associations de producteurs et des infrastructures de transformation et d'export agroalimentaires y sont présentes. Malgré une apparente atonie du développement agricole à Valle Azul, on y observe davantage d'installations de jeunes et les formes d'installation sont plus variées. Cette différence peut s'expliquer par la structure foncière. En effet, à Valle Azul, l'asentamiento le plus ancien, la structure égalitaire d'accès au foncier héritée de la réforme agraire n'est plus visible : elle a été remodelée par les différentes successions, divisions entre fratries, ventes et rachats réalisés en deux, voire trois générations. Aujourd'hui, de nombreuses terres sont à vendre et permettent aux jeunes de s'installer avec des structures foncières viables. À Futuro, le parcellaire de la réforme agraire est au contraire encore présent, puisque la génération des attributaires initiaux est encore sur place à travailler la terre. Cette structure foncière, faite de lot de 4 ha, ne permet pas de faire vivre deux familles (le père et le fils) et cadenasse le processus d'installation des jeunes agriculteurs (notamment la création). Au contraire, la structure foncière inégale et la diversification de l'activité économique à
Valle Azul (artisanat et développement du tourisme) permettent l'installation de jeunes agriculteurs (relève, création), contribuant ainsi au maintien d'une agriculture familiale.

\subsection{La dotation en capitaux}

Au-delà des caractéristiques des territoires qui influent sur la disponibilité et la distribution de capital physique (le foncier) et social (présence d'associations de producteurs), les dotations individuelles en capitaux financiers et humains et les trajectoires personnelles sont déterminantes pour comprendre la diversité des processus d'installation. En particulier, le capital humain lié à l'éducation et à l'apprentissage agricole semble un élément clé dans le processus d'installation : plus le jeune a un niveau scolaire élevé, plus il conçoit son exploitation comme une entreprise et est à même d'introduire des changements conséquents (Tab. 5 et 6 ).

Nous avons également observé une relation d'agrégation entre les différentes dotations individuelles de capitaux (Tab. 5) : plus un jeune est qualifié, plus ses capitaux financiers et sociaux sont également importants. Ces derniers facilitent la réalisation d'idées portées par les jeunes agriculteurs. Les jeunes agriculteurs qui ont appris ou perfectionné leurs connaissances agricoles hors du cercle familial apportent davantage de changement que ceux qui n'ont connu que l'exemple paternel.

Enfin, l'accès au foncier reste un élément crucial pour expliquer le type d'installation (Fig. 3 et Tab. 7). Cinq formes d'accès au foncier ont été identifiées : l'achat, la location, le prêt de terre, l'héritage acté et l'héritage promis. L'achat est très rare car l'apport financier nécessaire est souvent trop important pour les jeunes agriculteurs, qui sont en concurrence avec des acheteurs extérieurs à la recherche de résidences secondaires. La location permet de pallier le déficit de capital initial : c'est un effort important mais également la garantie d'une plus grande autonomie. Ainsi, alors que la reprise et la relève se font principalement via des héritages actés ou promis, la création fait appel à des formes foncières beaucoup plus variées et s'appuie majoritairement sur la location, le prêt et l'achat. 


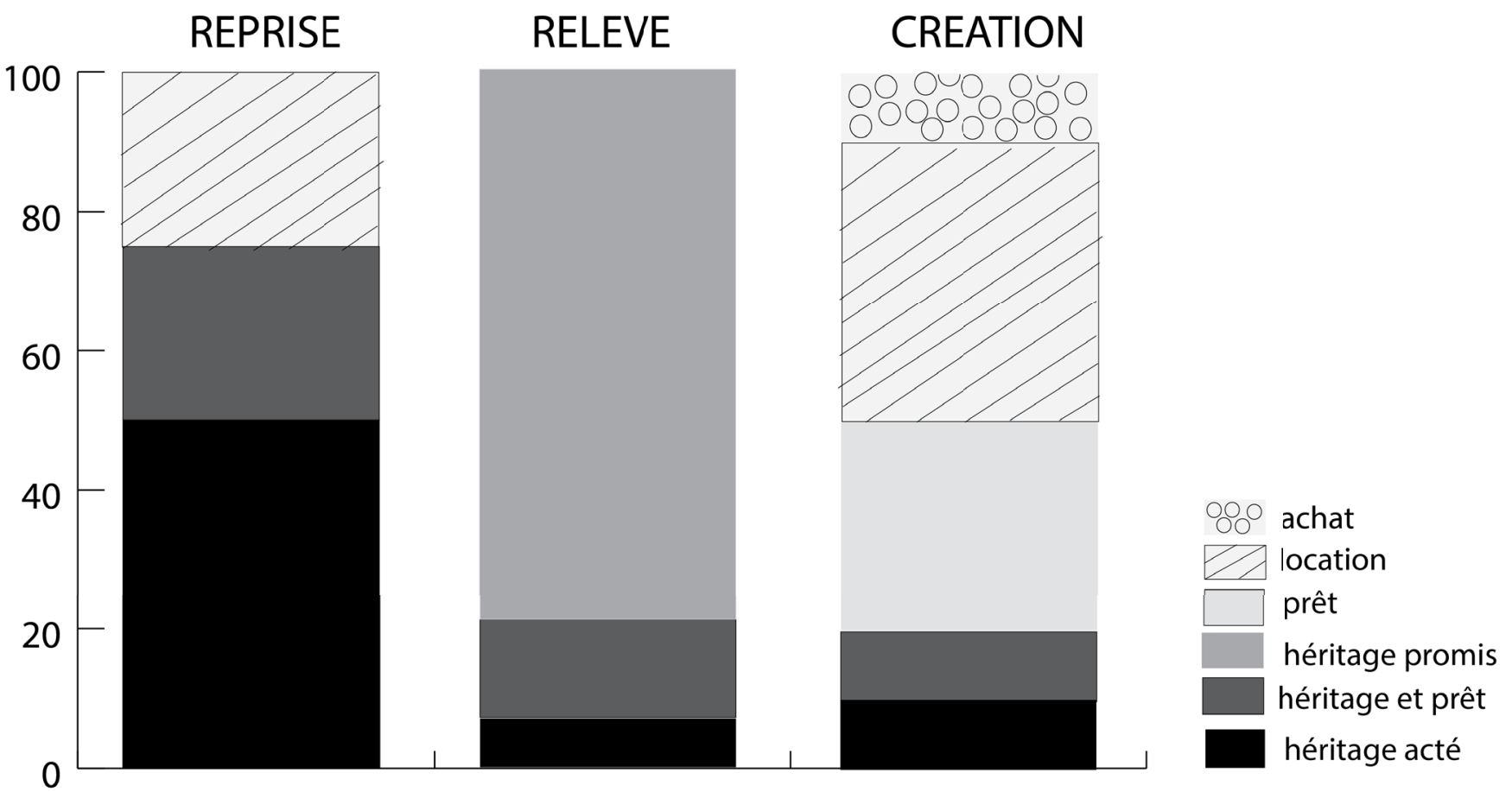

Fig. 3. Influence du capital foncier sur les différentes formes d'installation.

Fig. 3. How the property capital explains the different types of settling.

Tableau 7. Influence du foncier sur l'installation des jeunes agriculteurs.

Table 7. Influence of land structure on young farmers' settling.

\begin{tabular}{lll}
\hline & Valle Azul & Futuro \\
\hline Parcellaire égalitaire de la réforme encore visible & Non & Oui \\
Taille d'une exploitation & Entre 0,5 et 10 ha & 4 ha exclusivement \\
Disponibilité du foncier & Oui & Non \\
Nombre de jeunes agriculteurs rencontrés & 19 & 8 \\
Diversité des processus d'installation & Relève, reprise et création sont présentes & Aucun cas de création
\end{tabular}

Sources : entretiens, 2015.

\subsection{La relation au père}

La relation au père est un troisième élément clé pour expliquer les différents types d'installation observés. Nous pouvons caractériser la relation avec le père selon deux critères: le degré d'autonomie, qui caractérise la place accordée au jeune dans les choix décisionnels de l'exploitation, et le degré d'indépendance vis-à-vis du père en termes de capitaux (Tab. 6). C'est souvent pour ce gain d'autonomie et d'indépendance que le jeune agriculteur préfère s'installer seul en louant des terres (création ou reprise), plutôt que de travailler avec le père ou le beau-père sur une exploitation déjà existante où les perspectives d'évolution lui semblent limitées (relève).

Si dans les cas de reprise, le père s'efface complètement en déclarant que c'est bien son fils qui est à la tête de l'exploitation, les relations père-fils sont beaucoup plus complexes dans les cas de relève. Le degré d'autonomie laissé au fils par le père explique l'ampleur des changements que le jeune effectue : plus il est faible, moins il y a de changement. Le père reste le propriétaire du terrain sur lequel le fils s'est installé, ce qui lui confère une supériorité dans les décisions. Toutefois, dans ces exploitations où père et fils travaillent ensemble, le fils gagne en indépendance car il dispose d'une parcelle à lui, prêtée par le père, où il conduit de manière plus autonome ses activités (production de ses semences ou engraissement de veaux, par exemple).

\section{Discussion}

Nos résultats permettent de discuter trois dimensions de l'évolution et de la durabilité de l'agriculture familiale et de la place des jeunes dans l'agriculture des pays du Sud : l'émergence d'une nouvelle ruralité fondée sur la pluriactivité, l'importance de l'accessibilité des capitaux et des ressources dans les processus de renouveau générationnel, et l'adaptation des politiques agricoles et rurales pour faciliter la durabilité de l'agriculture familiale. 
Nos résultats confirment que l'agriculture familiale évolue vers davantage de pluriactivité (Dufumier, 2006). Cette évolution vers la pluriactivité est congruente avec le développement d'une nouvelle ruralité observable en Europe (Bonnal et al., 2004), en Afrique (Losch et al., 2013) et en Amérique latine (FAO, 2003), où l'agriculture reste un pilier du développement rural, mais de manière non exclusive, et où les activités non agricoles de service tournées vers les populations locales (commerces locaux) ou extérieures (tourisme) deviennent prépondérantes. Nos analyses confirment qu'une certaine reproduction sociale est à l'œuvre chez les agriculteurs familiaux (Bélières et al., 2013), mais que l'installation des jeunes agriculteurs peut être, dans certaines conditions (création, reprise), source importante de changements dans les systèmes de production.

Au-delà des motivations des jeunes à rester dans l'agriculture ou de leurs désirs d'émigration (Bednaríkova et al., 2016), notre analyse souligne que certaines conditions sont nécessaires pour que les jeunes puissent s'installer dans l'agriculture. À l'instar des études réalisées au Maghreb (Ameur et al., 2015 ; Amichi et al., 2015), la mise en place de projets agricoles par les jeunes devient effective si certaines conditions d'accès à des ressources et à des capitaux sont réunies. Notre étude confirme l'importance des contraintes d'accès à la terre et d'émancipation identifiées dans de multiples travaux (White, 2012 ; Bezu et Holden, 2014 ; Bouzidi et al., 2015b). Elle souligne aussi l'importance du facteur humain. Dans la lignée des travaux de Mesen (2009), elle montre qu'une relation existe entre la mise en place de projets nouveaux par les jeunes agriculteurs et leur capital humain en termes de niveau d'étude. Ainsi, les jeunes ayant fait des études sont à l'origine des changements les plus importants, qui semblent contribuer à la pérennité de l'agriculture familiale. Cette observation s'oppose à l'idée très répandue, pour le moins au Costa Rica, que les études sont néfastes aux campagnes puisqu'elles ne feraient qu'aspirer les jeunes diplômés dans les villes. Cependant, notre étude souligne que le niveau scolaire seul ne suffit pas pour expliquer les recompositions en cours et qu'il est nécessaire de considérer la distribution d'autres capitaux issus des trajectoires individuelles ou familiales, et des territoires. Sur notre terrain costaricain comme dans de nombreux pays du Sud (Bouzidi et al., 2015a), l'accès au foncier reste un enjeu majeur pour les jeunes agriculteurs. Les contrats fonciers formels sont rares et, dans une grande majorité des cas, les héritages sont simplement « promis », et de surcroît à toute la fratrie et à une échéance floue. Le maintien du contrôle du foncier par la génération précédente donne lieu à une situation précaire qui remet en cause la durabilité des exploitations des jeunes agriculteurs. $\mathrm{Si}$, pour contourner ces difficultés et avoir accès à la terre, certains jeunes agriculteurs marocains innovent techniquement ou développent un leadership en matière de développement (Bouzidi et al., 2015b), nous n'avons pu mettre en lumière une quelconque stratégie sur nos terrains. Toutefois, notre travail montre que cette contrainte liée au maintien d'un contrôle patriarcal sur les ressources n'est pas systématique. Le père, propriétaire du terrain, laisse un certain degré d'autonomie à son fils pour permettre des processus d'installation sous forme de relève ou de reprise. Si un lien intergénérationnel est nécessaire pour garantir la transmissibilité des exploitations (Landais, 1998), notre étude suggère qu'un moment de rupture avec le père est également nécessaire pour permettre une « émancipation » (Coradini, 2015 ; Hamamouche et al., 2015) qui permette aux jeunes de mettre en place des changements dans les exploitations. Au-delà du foncier, l'accès au capital financier constitue également une contrainte forte pour l'autonomisation des jeunes agriculteurs dans leur processus d'installation. Dans notre terrain costaricain, cette contrainte est partiellement levée grâce à des prêts auprès de membres de la famille ou d'amis proches. Toutefois, l'accès au microcrédit ou à des prêts à taux avantageux permettrait de faciliter les premiers investissements et donc l'indépendance des jeunes agriculteurs.

En termes de politique, au Costa Rica comme dans de nombreux pays africains (Chinsinga et Chasukwa, 2012), peu de choses sont faites pour faciliter l'accès à ces différents capitaux ou leur sécurisation pour les jeunes agriculteurs. Nous rejoignons ainsi Ameur et al. (2015) sur le fait que les pouvoirs publics doivent reconnaître le rôle des jeunes agriculteurs dans le développement agricole et territorial et faciliter les processus de succession en cours dans les exploitations familiales. Audelà de politiques différenciées pour les agricultures familiales (Sabourin et al., 2014), plusieurs pistes spécifiques peuvent être proposées pour aider le renouveau générationnel des agricultures familiales dans les pays du Sud, en s'inspirant des instruments de politiques développés en Europe (Mundler et Ponchelet, 1999 ; Le Blanc, 2011). Au-delà des efforts de formation professionnelle qui constituent souvent l'élément principal, voire exclusif, des politiques pour l'intégration des jeunes ruraux dans l'agriculture dans les pays du Sud, il s'agirait de faciliter leur accès aux facteurs de production :

- en encourageant la transmission du foncier sous forme d'héritage acté grâce à une simplification des procédures de transmission, en réduisant les frais de succession (politique fiscale) et en facilitant l'accès à la retraite des générations précédentes (Bika, 2007) ;

- en proposant aux jeunes agriculteurs des financements conséquents et à faible taux (tels que les DJA) pour faciliter les processus de reprise ou de création, ou des microcrédits pour favoriser les relèves dans la perspective d'autonomisation vers des reprises et des créations;

- en favorisant la création d'organisations de jeunes producteurs ou leur insertion dans les organisations existantes, afin qu'ils bénéficient d'un ensemble de services coordonnés (Le Coq et al., 2012), facilitant la durabilité de leurs projets.

\section{Conclusion}

L'installation de jeunes agriculteurs participe aux recompositions de l'agriculture familiale et de la ruralité. L'identification de trois types d'installation, la relève, la reprise et la création, permet d'éclairer la diversité des processus de renouvellement générationnel et de changements agricoles en cours. Selon ces types d'installation, les modifications apportées par les jeunes agriculteurs sont extrêmement variables. Alors que les créations sont associées à des changements radicaux, la reprise et la relève, qui sont les types les plus courants, conduisent à des changements bien plus limités en termes de systèmes de production et d'activités. Néanmoins, avec une majorité de jeunes agriculteurs pratiquant 
l'agriculture seulement à temps partiel, les processus d'installation préfigurent un nouveau modèle pour l'agriculture familiale : une agriculture familiale pluriactive basée sur des activités non agricoles.

La diversité des types d'installation s'explique par la possibilité pour les jeunes agriculteurs d'accéder aux capitaux, notamment fonciers, humains (éducation) et financiers, en lien avec les trajectoires des territoires et les relations avec la génération précédente (notamment les pères).

Alors que les politiques d'accompagnement à l'installation des jeunes agriculteurs dans les pays du Sud sont souvent inexistantes, la caractérisation de différents types d'installation et la compréhension des facteurs explicatifs qui président à cette diversité au Costa Rica, et plus généralement dans les pays du Sud, permettent d'envisager des politiques différenciées et adaptées aux conditions de ces nouveaux exploitants. Ces dernières devraient notamment s'attacher à favoriser les cas de reprise, aujourd'hui si rares, tout comme ceux de création en promouvant la formation professionnelle agricole, mais également et surtout en mettant en place des instruments de politiques encourageant la transmission du foncier depuis les générations précédentes (politique fiscale incitative, aide à la cessation d'activité des parents) et permettant aux jeunes agriculteurs d'investir dans et hors de l'agriculture via des dotations ou des (micro)crédits spécifiques. Ce faisant, le passage de relais entre les générations dans la petite agriculture familiale serait assuré et contribuerait à maintenir des territoires ruraux vivants et attractifs.

\section{Références}

Aggelopoulos S, Arabatzis G. 2010. European union young farmers program: a Greek case study. New Medit 9(2): 50-55.

Ameur F, Quarouch H, Dionnet M, Lejars C, Kuper M. 2015. Outiller un débat sur le rôle des jeunes agriculteurs dans une agriculture en transition dans le Saïss (Maroc). Cahiers Agricultures 24: $363-371$.

Amichi H, Kadiri Z, Bouarfa S, Kuper M. 2015. Une génération en quête d'opportunités et de reconnaissance : les jeunes ruraux et leurs trajectoires innovantes dans l'agriculture irriguée au Maghreb. Cahiers Agricultures 24: 323-329.

Atkinson R, Flint J. 2010. Accessing hidden and hard-to-reach populations: snowball research strategies. Social Research Update 28(1): 93-108.

Bednaríkova Z, Bavorova M, Ponkina E. 2016. Migration motivation of agriculturally educated rural youth: the case of Russian Siberia. Journal of Rural Studies 45: 99-111.

Bélières JF, Bonnal P, Bosc PM, Losch B, Marzin J, Sourisseau JM. 2013. Les agricultures familiales du monde. Définitions, contributions et politiques publiques. France : AFD-Cirad.

Bezu S, Holden S. 2014. Are rural youth in Ethiopia abandoning agriculture? World Development 64: 259-272.

Bika Z. 2007. The territorial impact of the farmers' early retirement scheme. Sociologia Ruralis 47(3): 246-272.

Bonnal P, Losch B, Bosc P.M, Diaz J. 2004. Multifonctionnalité de l'agriculture et nouvelle ruralité. Une mise en perspective sur deux démarches de refondation des politiques publiques. In : Bonnal P, ed. Politiques publiques et comparaison internationale. France: CEMAGREF, p. 61-81.
Bourdieu P. 1984. Questions de sociologie. France : Éditions de Minuit.

Bouzidi Z, Faysse N, Kuper M, Billaud JP. 2015a. Les projets des jeunes ruraux : des stratégies diversifiées pour accéder au foncier et obtenir l'appui de l'État. Alternatives Rurales, hors-série.

Bouzidi Z, Kuper M, Billaud JP, Faysse N. 2015b. Mobiliser des ressources techniques et sociales pour s'installer: stratégies des jeunes ruraux au Maroc. Cahiers Agricultures 24: 420-427.

Chambers R, Conway G. 1991. Sustainable rural livelihoods: practical concepts for the 21st century. IDS Discussion Paper 296, $33 \mathrm{p}$.

Chinsinga B, Chasukwa M. 2012. Youth, agriculture and land grabs in Malawi. IDS Bulletin 6(43): 67-77.

Coradini L. 2015. Young farmers and generational reproduction in family farming: a case study of young people residing in the municipality of Faxinal Soturno - Brazil. Mundo Agrario 16(33): $1-12$.

Costa F, Ralisch R. 2013. A juventude rural do assentamento florestan fernandes no município de Florestópolis. Revista de Economia e Sociologia Rural 3(51): 415-432.

Davis A. 2008. Declining youth in-migration in rural Western Australia: the role of perceptions of rural employment and lifestyle opportunities. Geographical Research 46(2): 162-171.

Desmulier D, Gasselin P. 2015. La quadrature des agricultures familiales : instruire et dépasser les controverses. Revue Tiers Monde 221: 129-146.

Duby G, Gervais M, Tavernier Y, Jolivet M. 1992. Histoire de la France rurale, tome 4 : depuis 1914. Paris : Seuil.

Dufumier M. 2006. Diversité des exploitations agricoles et pluriactivité des agriculteurs dans le Tiers Monde. Cahiers Agricultures 15(6): 584-588.

FAO. 2003. La nueva ruralidad en Europa y su interés para América Latina. Rome: FAO.

FAO, CTA, FIDA. 2014. Les jeunes et l'agriculture : principaux enjeux et solutions concrètes. Rome : FAO.

Faure G, Samper M. 2005. Vingt ans d'ouverture économique : l'avenir compromis de l'agriculture familiale au Nord du Costa Rica. Revue Tiers Monde 46: 581-602.

Faure G, Maître d'Hôtel E, Le Coq JF. 2010. Les organisations de producteurs agricoles sont-elles garantes d'un meilleur accès des producteurs aux marchés agricoles ? Enseignements d'une étude menée au Costa Rica. Revue internationale de l'économie sociale, RECMA 316: 85-105.

Hamamouche MF, Kuper M, Lejars C. 2015. Emancipation des jeunes des oasis du Sahara algérien par le déverrouillage de l'accès à la terre et à l'eau. Cahiers Agricultures 24: 412-419.

Lacombe P. 2016. L'agriculture familiale : rengaine ou nouveauté ? Natures Sciences Sociétés 24: 123-135.

Landais E. 1998. Agriculture durable: les fondements d'un nouveau contrat social? Le Courrier de l'environnement de l'INRA, Paris : Institut national de la recherche agronomique Délégation permanente à l'environnement, 33 (33), p. 5-22.

Le Coq JF, Faure G, Saenz F. 2012. Les organisations de producteurs dans le système de services agricoles au Costa Rica. Economie rurale 330-331: 175-190.

Le Blanc J. 2011. Installation agricole : nouveaux profils, nouvel accompagnement. Pour 5(12): 137-143.

Losch B, Magrin G, Imbernon J. 2013. Une nouvelle ruralité émergente - Regards croisés sur les transformations rurales africaines. France : Cirad.

Mesen R. 2009. La situación educativa y ocupacional de los jóvenes rurales y su implicaciónpara la economia familiar, la sostenibilidad del agrosistema y sus proyectos de vida. Estudio de caso en Tierra 
Blanca de Cartago. Costa Rica. Thèse de doctorat, université Rodrigo Facio (Costa Rica).

Muhammad-Lawal A, Omotesho O, Falola A. 2003. Technical efficiency of youth participation in agriculture: a case study of the youth in agriculture programme in Ondo state. Nigerian Journal of Agriculture, Food and Environment 5(20): 20-26.

Mundler P, Ponchelet D. 1999. Agriculture et mobilité sociale. Ces agriculteurs venus d'ailleurs. Economie rurale 253(1): 21-27.

Purseigle F. 2004. Du texte aux variables. Contribution de l'analyse textuelle à la compréhension de l'engagement professionnel des jeunes agriculteurs. Ruralia. Sciences sociales et mondes ruraux contemporains 14: 1-12.

Quarouch H, Lejars C, Kuper M. 2015. Recevoir la parole des institutions et la leur retourner : parcours agricoles de jeunes ruraux diplômés-chômeurs dans le Saïss-Maroc. Cahiers Agricultures 24: 349-355.

Rémy J. 1997. Les sans-dot de l'agriculture : faut-il aider les installations sans aide ? Économie rurale 238(1): 33-37.
Ruben R, Saenz F. 2008. Farmers, markets and contracts: chain integration of small holder producers in Costa Rica. European Review of Latin American and Caribbean Studies 85: 61-81.

Sabourin É, Marzin J, Le Coq JF, Massardier G, Fréguin-Gresh S, Samper M, et al. 2014. Agricultures familiales en Amérique latine. Émergence, avancées et limites des politiques ciblées. Revue Tiers Monde 4: 23-41.

Salazar JA, Le Coq JF, Saenz F. 2012. Medios de vida de las familias dedicadas a actividades agrícolas y ganaderas en el cantón de Guatuso, Alajuela. Ciencias económicas 30: 201-217.

Sourisseau JM. 2014. Agricultures familiales et mondes à venir. France : Éditions Quae.

Sourisseau JM, Bosc PM, Fréguin-Gresh S, Bélières JF, Bonnal P, Le Coq JF, et al. 2012. Les modèles familiaux de production agricole en question. Comprendre leur diversité et leur fonctionnement. Autrepart 62: 159-181.

White B. 2012. Agriculture and the generation problem: rural youth, employment and the future of farming. IDS Bulletin, 43 (6) :9-19, special issue: Young people and agriculture in Africa.

Citation de l'article : Parquet M, Le Coq J-F. 2017. Installation des jeunes agriculteurs et pluriactivité au Costa Rica. Cah. Agric. $26: 15004$. 